\title{
Tailored stereotactic radiotherapy technique using deep inspiration breath-hold to reduce stomach dose for cardiac radioablation
}

\author{
Myung-Jin Cha', Phillip S. Cuculich², Clifford G. Robinson ${ }^{3}$, Ji Hyun Chang ${ }^{4}$ \\ 'Cardiovascular Division, Department of Internal Medicine, Seoul National University Hospital, Seoul, Korea \\ ${ }^{2}$ Cardiovascular Division, Department of Internal Medicine, Washington University, St Louis, MO, USA \\ ${ }^{3}$ Department of Radiation Oncology, Washington University, St Louis, MO, USA \\ ${ }^{4}$ Department of Radiation Oncology, Seoul National University Hospital, Seoul, Korea
}

Received: February 22, 2021

Revised: April 12, 2021

Accepted: April 14, 2021

Correspondence:

Ji Hyun Chang

Department of Radiation Oncology,

Seoul National University College of

Medicine, 101 Daehak-ro, Jongno-gu,

Seoul 03080, Korea.

Tel: +82-2-2072-4940

E-mail: jh.chang@snu.ac.kr

ORCID:

https://orcid.org/0000-0001-5921-5522
Purpose: To provide a new insight on a novel safe cardiac radioablation using deep inspiration breath-hold (DIBH) to reduce gastrointestinal dose.

Materials and Methods: For treating incessant ventricular tachycardia (VT) originated from left ventricle inferior scar abutting the stomach, a target delineation and treatment planning for cardiac radioablation was performed. With four different computed tomography (CT) scan protocols-DIBH, full expiration breath-hold, four-dimensional (4D) CT without and with abdominal compression, the distances between the target and the stomach were compared.

Results: Among the protocols, the CT scan with DIBH showed largest distance between the target and the stomach and selected for the treatment planning. The prescribed dose was $25 \mathrm{~Gy}$ in a single fraction, and satisfactory dosimetric parameters were achieved with the DIBH. The patient was successfully treated with the DIBH, and experienced no acute toxicity.

Conclusion: To gain the best benefit from cardiac radioablation, understanding the possible toxicity in the adjacent organs is crucial. By moving the heart with thoraco-diaphragmatic movement by $\mathrm{DIBH}$, the target could be physically separated from the stomach.

Keywords: Radiotherapy, Ventricular tachycardia, Deep inspiration breath-hold

\section{Introduction}

Noninvasive cardiac radioablation, delivered with stereotactic body radiotherapy (SBRT), was recently introduced as a treatment option for life threatening refractory ventricular tachycardia (VT) [1,2]. Depending on location of the target within the ventricle, a variety of organs-at-risk such as the esophagus, stomach, and bowel could be exposed to high doses of radiation. Although few high-grade late complications have been described [3], one patient on the ENCORE-VT trial (electrophysiology-guided noninvasive cardiac radioablation for ventricular tachycardia) developed a grade 3 gastropericardial fistula 2-year after treatment [4], and another patient treated by Ostrava group developed a grade 5 esophageal fis- tula [5]. Therefore, the radiation risk of luminal internal organs in particular should be considered during treatment planning.

Cardiac radioablation targets along the inferior wall of the ventricle represent a particular emerging challenge due to their inherent proximity to structures such as the esophagus, stomach, and bowel. At present, no specific techniques have been described to improve dosimetry for these complex targets. Furthermore, the heart does not stick to adjacent internal organs, but moves and rotates with thoraco-diaphragmatic movement. Herein, we report a successful cardiac radioablation using the deep inspiration breathhold (DIBH) technique to minimize toxicity to the stomach during cardiac radioablation. 


\section{Materials and Methods}

\section{Patient information and target definition}

With Institutional Review Board approval of Seoul National University Hospital (No. 2011-151-1175), cardiac radioablation was considered for the medically intractable VT because catheter ablation was contraindicated due to the previous cardiac valve surgeries. Informed consent of the patient for radiotherapy under clinical trial (blinded) [6] was obtained. The detailed patient information was described in Supplement A.

Based on the 12-leads electrocardiography (Fig. 1), the VT seemed to originate from the mid-inferior left ventricle (LV). Cardiac magnetic resonance imaging (MRI) confirmed a transmural and subendocardial delayed enhancement in the mid-inferior and inferolateral segments, indicating myocardial scarring (Fig. 2).

\section{CT simulation and target delineation}

The patient underwent the four different computed tomography (CT) scans with slice thickness of $2 \mathrm{~mm}$ using the Brilliance CT Big Bore system (Phillips, Cleveland, OH, USA): (1) DIBH, (2) full expiration breath-hold, (3) four-dimensional (4D) CT without abdominal compression, and (4) 4D-CT with abdominal compression. The respiration-gated 10 phase 4D-CT images were acquired using the
Real-time Position Management system (RPM; Varian Medical Systems, Palo Alto, CA, USA). The VT focus shown in electrocardiography and cardiac MRI was delineated as gross target volume (GTV). The margin for clinical target volume (CTV) was $1 \mathrm{~cm}$, and $0.5 \mathrm{~cm}$ was additionally expanded for planning target volume (PTV). Generally, electrophysiological mapping could provide more accurate information on the location of the VT focus, however in this case, invasive 3D mapping could not be performed due to patient's previous surgical history. Therefore, the CTV was extended to account for possible spread of myocardial scar at border lesions, which might have not been visualized on MRI. To further limit the radiation dose to the stomach, the stomach planning organ-at-risk volume (PRV) was determined as stomach plus $0.5 \mathrm{~cm}$. Of the four scans, the largest distance between the stomach and LV scar was achieved on the DIBH scan (Fig. 3). The closest distance between the stomach and the PTV was $0.67 \mathrm{~cm}$ in DIBH, $0.49 \mathrm{~cm}$ in average image of 4D CT without abdominal compression and $0 \mathrm{~cm}$ (overlapped) in full expiration breath-hold and average image of 4D CT with abdominal compression. Meanwhile, GTV, CTV, PTV, stomach, and stomach PRV delineation on the CT scans with full expiration, average intensity 4D CT image (4DCT-AVG) with abdominal compression, and 4DCT-AVG without abdominal compression showed abutment or overlap of PTV and stomach PRV. Therefore, treatment

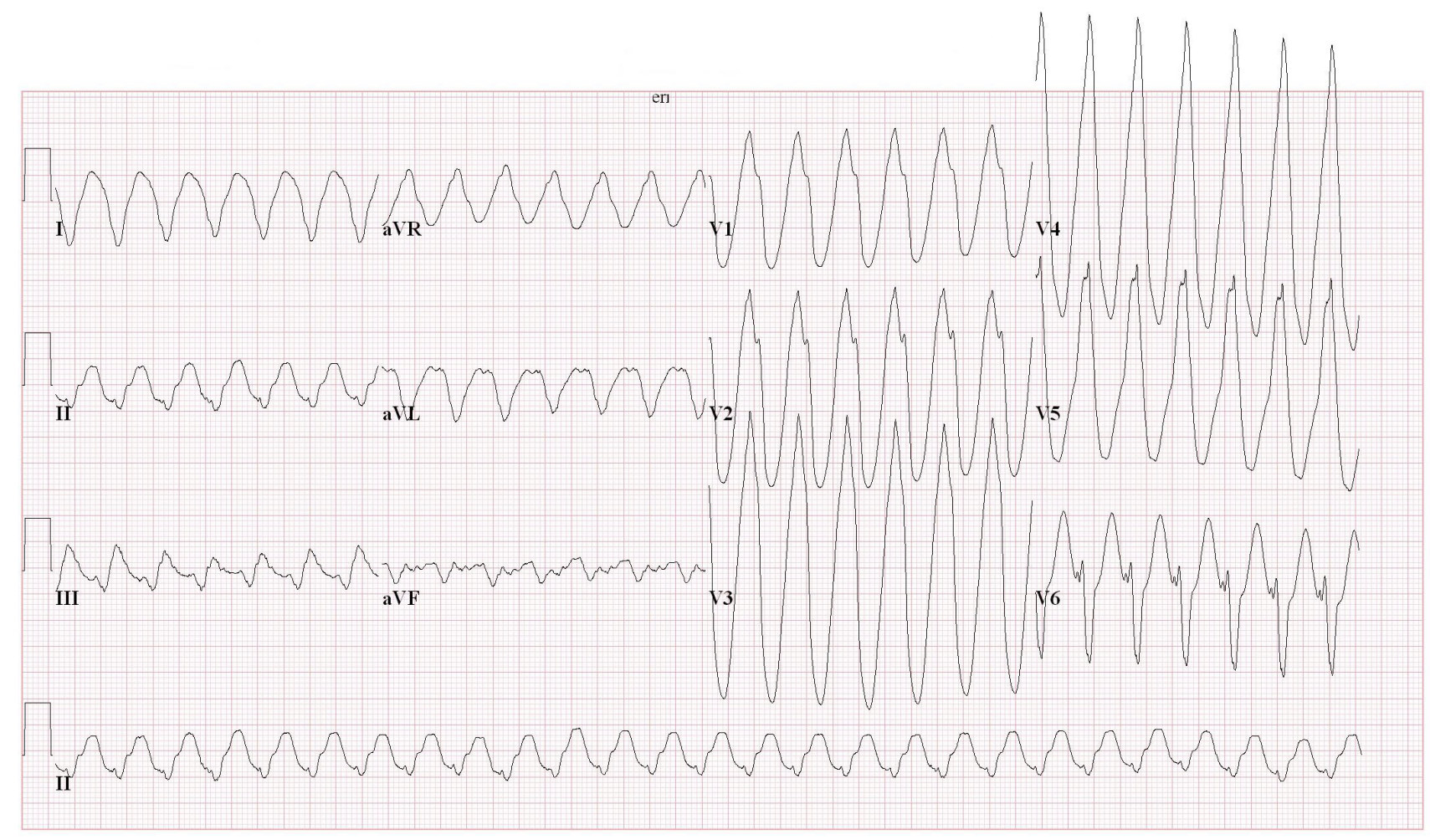

$25 \mathrm{~mm} / \mathrm{s} \quad 10 \mathrm{~mm} / \mathrm{mV} \quad 40 \mathrm{~Hz}$

Fig. 1. Electrocardiography of ventricular tachycardia. 


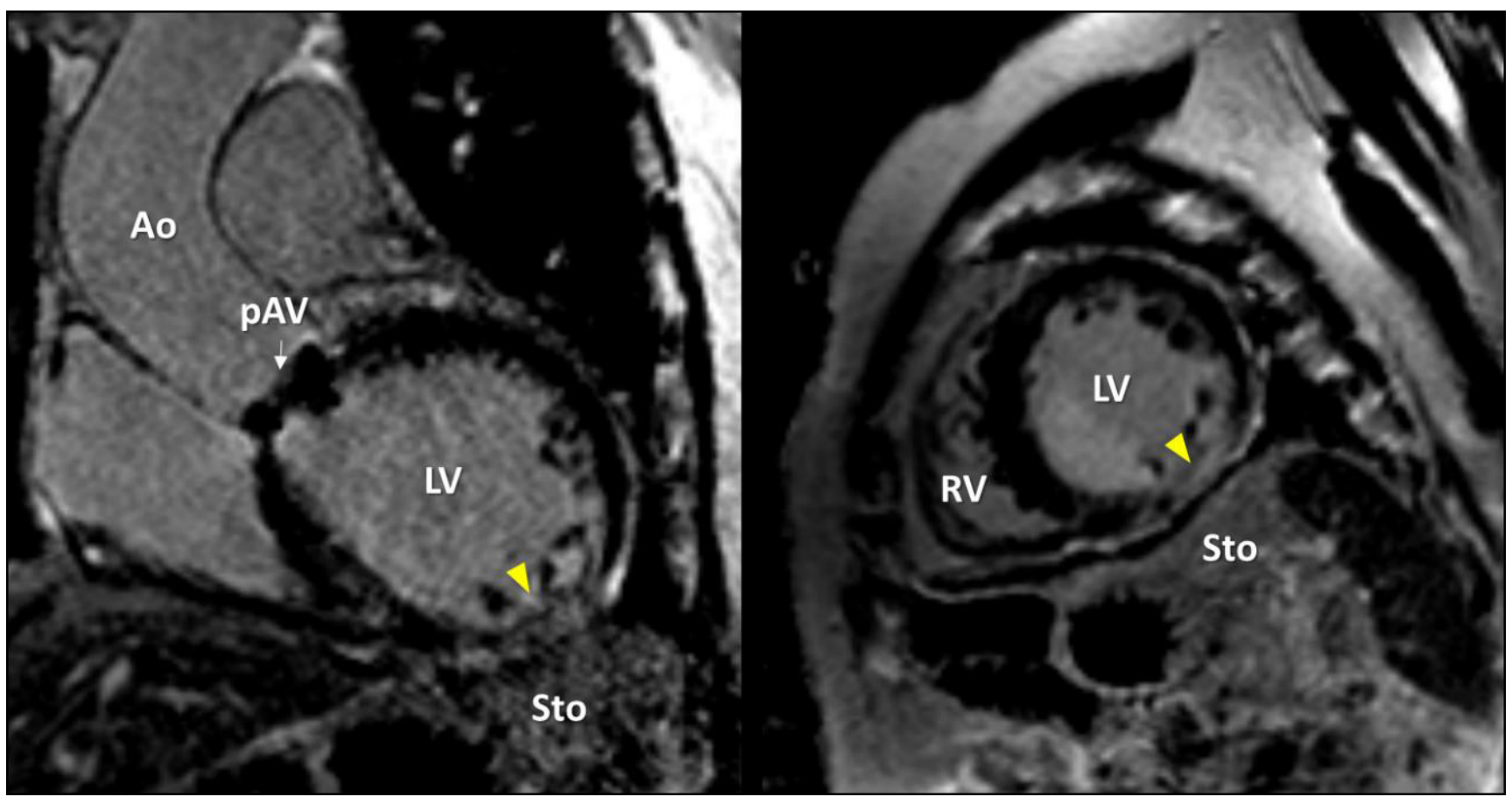

Fig. 2. Myocardial scar on cardiac magnetic resonance imaging. Ao, aorta; pAV, prosthetic aortic valve; LV, left ventricle; RV, right ventricle; Sto, stomach.
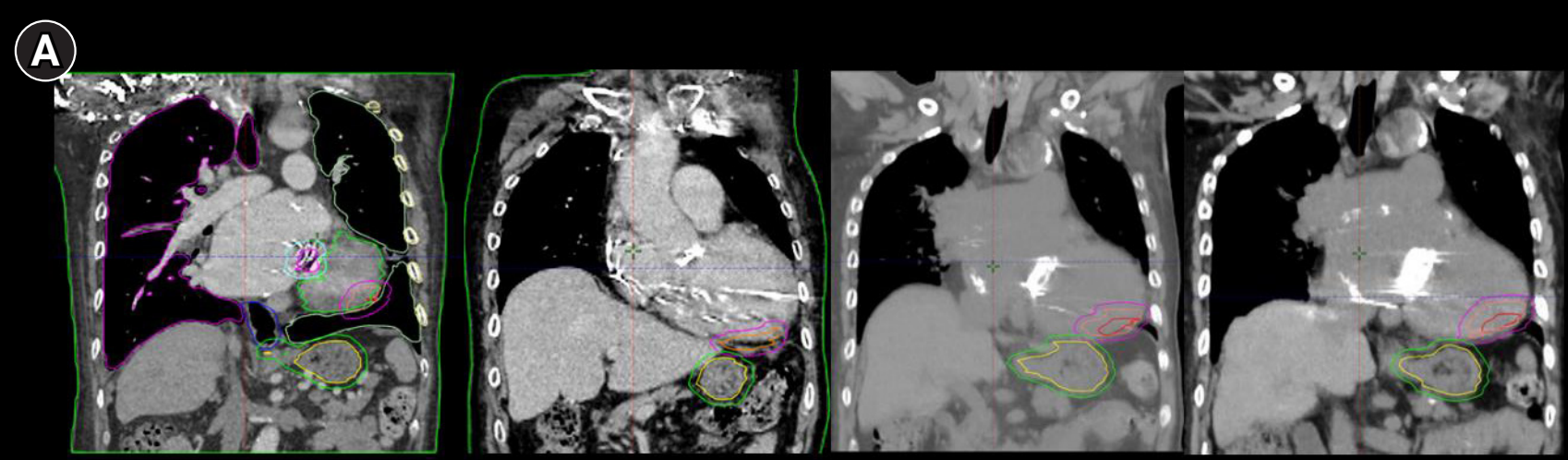

(B)
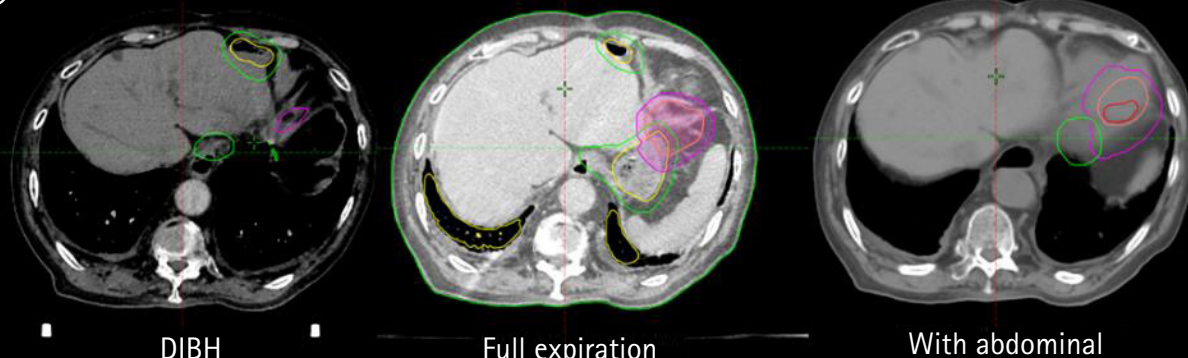

Full expiration

With abdominal compression

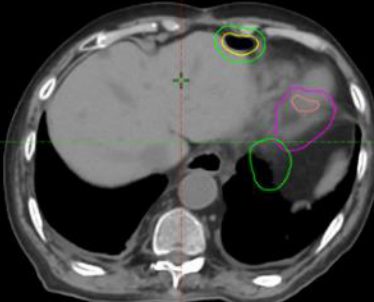

With abdominal compression

Fig. 3. (A) Representative coronal images showing largest distance between the clinical target volume (orange), planning target volume (purple) and the stomach (yellow) and stomach planning organs-at-risk volume (light green) in deep inspiration breath-hold (DIBH, left) and full expiration breath-hold (right). (B) Axial images showing shortest distance in DIBH, full expiration breath-hold, average image of four-dimensional computed tomography with and without abdominal compression. 
planning was performed using the DIBH scan.

\section{VMAT planning}

The prescribed dose to the PTV was $25 \mathrm{~Gy}$ in a single fraction. The Eclipse system (Varian Medical Systems) was used for treatment planning, and volumetric modulated arc therapy (VMAT) planning was performed using three half arcs with a 10-MV flattening filter-free beam (Fig. 4). The plan was optimized to achieve a PTV coverage of $\mathrm{V}_{98 \%}=100 \%$ while applying maximum dose constraint to stomach PRV of 10 Gy. The maximum dose within the PTV was $113 \%$ of the prescribed dose. The actual maximum dose to the stomach PRV and stomach was 14.6 and $9.8 \mathrm{~Gy}$, respectively.

\section{Results}

\section{Cardiac radioablation using DIBH}

The Varian TrueBeam linear accelerator (Varian Medical Systems) was used for cardiac radioablation. Prior to on-board cone-beam
CT (CBCT) acquisition, fluoroscopy guidance tracking using implantable cardioverter defibrillator (ICD) lead tip and prosthetic valve contour projection was performed. As the patient could tolerate a breath-hold state for approximately 20 seconds, CBCT was performed with three DIBHs, and the treatment was performed with multiple DIBHs. CBCT matching was performed using contours of the cardiac outline and adjacent organs, including the bronchus and bones. The patient could successfully reproduce DIBH by coaching. Under breath-hold mode, the beam was manually turned on only when the inspiration level was achieved (Fig. 5). Total monitor unit (MU) was $2175+3171+3431 \mathrm{MU}$ and delivered at 600 $\mathrm{MU} / \mathrm{min}$. The total beam-on time was 15.2 minutes.

The patient kept fasting before radioablation and no preventive prescription was given to the patient.

\section{Outcome}

Within 2-day after radiotherapy, VT burden decreased by $46.4 \%$ except single ICD therapy. Afterwards, the patient experienced no

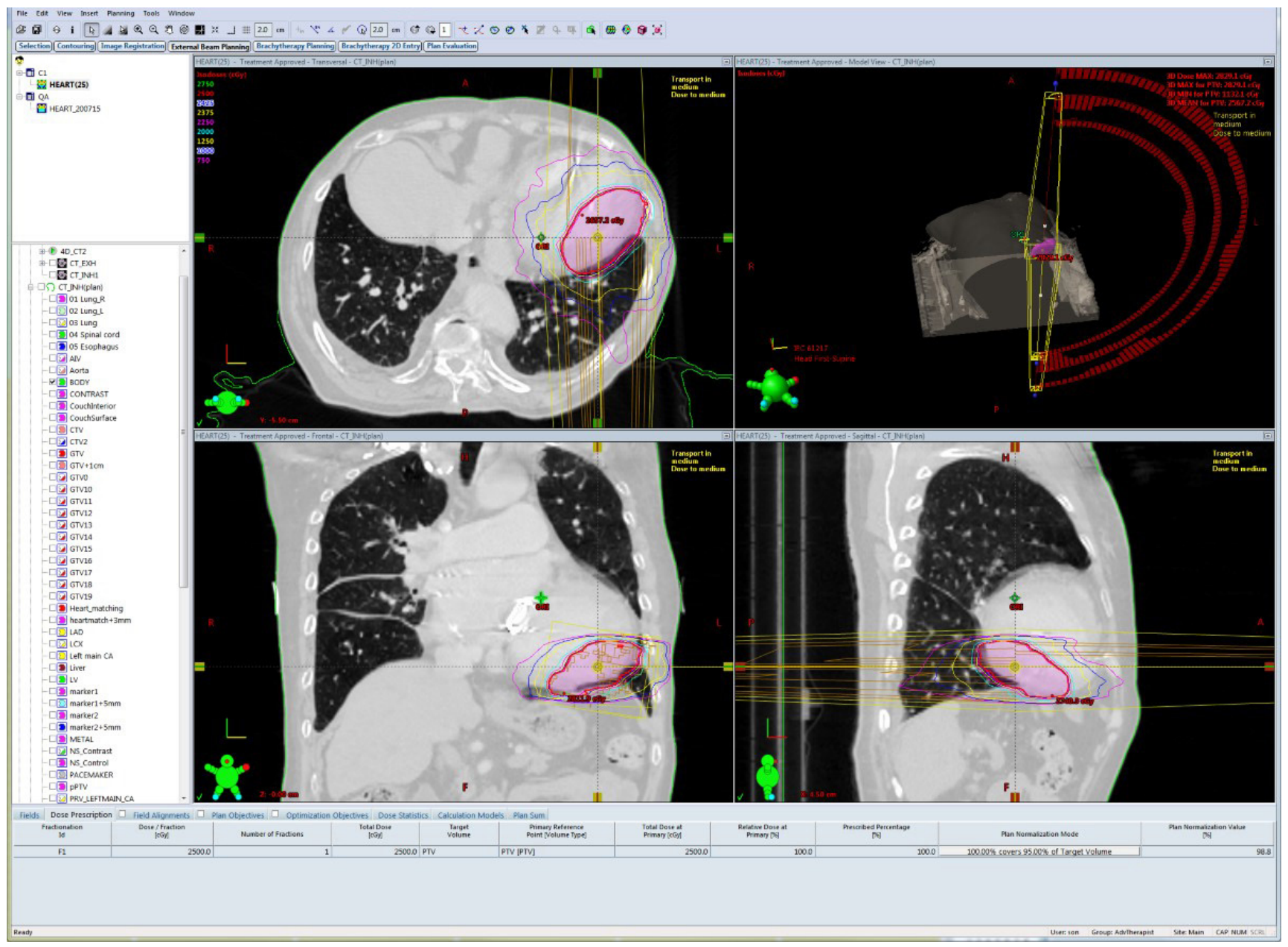

Fig. 4. Volumetric modulated arc therapy planning using deep inspiration breath-hold computed tomography. 


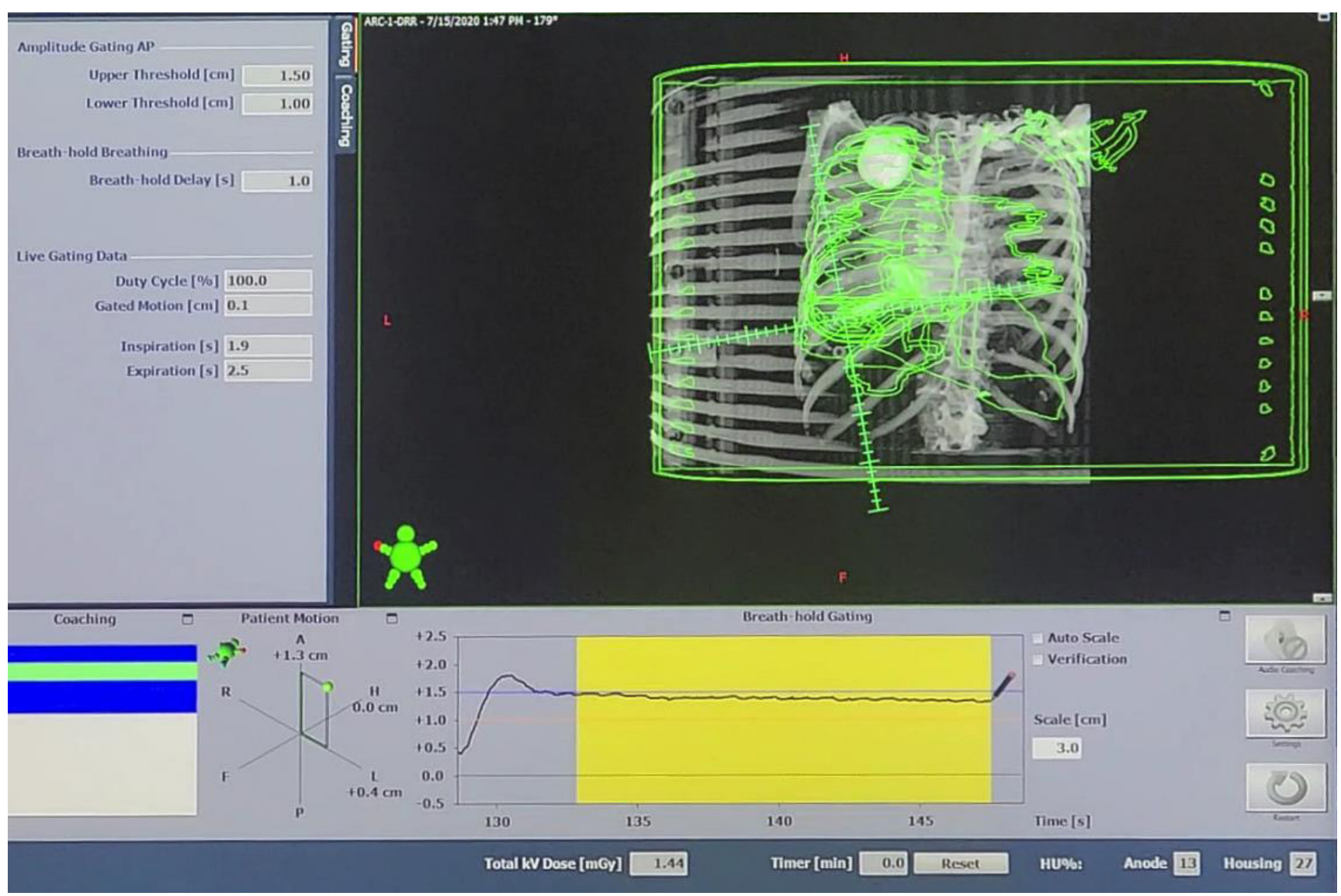

Fig. 5. Deep inspiration breath-hold using Real-time Position Management system. The yellow box represents beam-on state.

VT event or abdominal discomfort. Esophagogastroduodenoscopy is not planned for routine surveillance due to the patient's medical comorbidities and will be performed when the patient has related symptoms.

\section{Discussion and Conclusion}

To the best of our knowledge, this is the first report to show that gastrointestinal irradiation could be minimized during cardiac radioablation in the inferior LV using a tailored radiotherapy with $\mathrm{DIBH}$. We tried DIBH to overcome the possibility of a gastropericardial fistula during irradiation reported from ENCORE-VT trial $[1,4]$. In this case, the target was the inferior LV (the portion of the heart closest to the stomach) [7]. The Ostrava group reported one grade 5 esophagi-pericardial fistula in abstract form, therefore the detailed information would be available later [5].

Several studies have attempted to reduce the heart dose by DIBH in cases of gastric lymphoma and breast cancer [8-10]. The DIBH technique is gated irradiation during deep inspiration, investigated by several studies, mostly focused on reducing the dose to the heart. In this case, we conversely used DIBH to reduce the radiation dose to the stomach for cardiac radioablation. During deep inspiration, the heart descends with the diaphragm and becomes more elongated. Additionally, an inspiratory intrathoracic pressure decrease causes a LV volume decrease [11]. Furthermore, the heart rotates medially because part of the pericardium attaches to the diaphragm [12]. Consequently, the LV is physically separated from the stomach, which moves downward with the diaphragm during deep inspiration.

RT-related gastrointestinal toxicities include bleeding, ulceration, perforation, or fistula [13]. The tolerance dose for grade $\geq 2$ gastroduodenal toxicity in SBRT is variable among studies [14-17]; however, most of the studies have applied fractionated SBRT. The Quantitative Analyses of Normal Tissue Effects in the Clinic report recommended $V_{22.5}$ to be minimized and ideally constrained to $<4 \%$ [18]. This constraint was used for a study in Stanford University, and the authors reported a dosimetric model of duodenal toxicity after a single-fraction SBRT for pancreatic cancer and reported that $V_{15} \geq 9.1 \mathrm{~cm}^{3}, V_{20} \geq 3.3 \mathrm{~cm}^{3}$, and $D_{\max } \geq 23$ Gy were related to increased duodenal toxicity [15]. According to these data, our dosimetric parameters satisfied dose constraints far below the cutoff values. 
The possible short-term and long-term toxicity data should be monitored after cardiac radioablation. Furthermore, it is important to predict and prevent possible complications depending on the target location. In preclinical data, bronchomediastinal fistula, conduction block, or sudden death have been reported [19-21]. In clinical studies, some patients experienced radiation pneumonitis, pericarditis, or pericardial effusion that were mostly managed with observation or medications, except gastrointestinal fistula. Overall, most patients showed a good safety profile associated with irradiation [22]. Therefore, cardiac radioablation is potentially safe for intractable VT patients. To improve the safety of cardiac radioablation, using DIBH in VT patients would be applicable in patients with the target near the stomach, which could be mostly inferior LV, but it would depend on each patient's anatomical differences. It is essential to consider tailored radiotherapy techniques in each case and the DIBH technique would be one option for securing the distance between the heart and stomach.

In conclusion, to gain the best benefit from cardiac radioablation for fatal VTs, understanding the possible toxicity in adjacent organs is crucial. Successful treatment could be achieved by applying the DIBH technique while saving the stomach from excessive radiation, which could have caused serious complications.

\section{Conflict of Interest}

Dr. Cuculich and Dr. Robinson reports grants and personal fees from Varian, outside the submitted work. In addition, Dr. Robinson has a patent, "Noninvasive imaging and treatment system for cardiac arrhythmias (W02017078757A1)" licensed to Varian, and a patent US Provisional Application No. 62/598,162 entitled "System and method for determining segments for ablation," licensed to Varian. Otherwise, no potential conflict of interest relevant to this article was reported.

\section{Acknowledgments}

This work was supported by National Research Foundation of Korea grant funded by the Korean government (No. NRF-2018M2A2B3A01070410) and KOSRO Young Investigator Fund to Ji Hyun Chang and Myung-Jin Cha (No. NRF-2020R1A2C1013832).

\section{Supplementary Materials}

Supplementary materials can be found via https://doi.org/10.3857/ roj.2021.00276

\section{References}

1. Robinson CG, Samson PP, Moore KM, et al. Phase I/II trial of electrophysiology-guided noninvasive cardiac radioablation for ventricular tachycardia. Circulation 2019;139:313-21.

2. Cuculich PS, Schill MR, Kashani $R$, et al. Noninvasive cardiac radiation for ablation of ventricular tachycardia. N Engl J Med 2017;377:2325-36.

3. van der Ree MH, Blanck O, Limpens J, et al. Cardiac radioablation: a systematic review. Heart Rhythm 2020;17:1381-92.

4. Robinson CG, Samson P, Moore KM, et al. Longer term results from a phase I/II study of EP-guided noninvasive cardiac radioablation for treatment of ventricular tachycardia (ENCORE-VT). Int J Radiat Oncol Biol Phys 2009;105(3 Suppl):E682.

5. Cvek J, Sramko M, Knybel L, Hecko J, Kautzner J. Long-term toxicity of radiosurgery for ablation of ventricular tachycardia. Int J Radiat Oncol Biol Phys 2020;108(3 Suppl):E230-E231.

6. The Clinical Research Information Service. Clinical feasibility study of Noninvasive Cardiac Radio-ablation for Treatment of Ventricular tachycardia (KCT0004302) [Internet]. Osong, Korea: National Center for Medical Information and Knowledge; 2019 [cited 2021 May 31]. Available from: https://cris.nih.go.kr/cris/ search/detailSearch.do?all_type $=$ Y\&tsearch_page $=$ LEtpageSize $=10$ tpage $=1$ \&tseq $=14745$

7. Samson P, Hugo G, Moore K, Knutson N, Cuculich P, Robinson C. Noninvasive cardiac radioablation at Washington University: past, present, future directions for the treatment of VT. Appl Rad Oncol 2020;9:10-4.

8. Christopherson KM, Gunther JR, Fang P, et al. Decreased heart dose with deep inspiration breath hold for the treatment of gastric lymphoma with IMRT. Clin Transl Radiat Oncol 2020;24:7982.

9. Choi SH, Park SH, Lee JJ, Baek JG, Kim JS, Yoon HI. Combining deep-inspiration breath hold and intensity-modulated radiotherapy for gastric mucosa-associated lymphoid tissue lymphoma: Dosimetric evaluation using comprehensive plan quality indices. Radiat Oncol 2019;14:59.

10. Zhao F, Shen J, Lu Z, et al. Abdominal DIBH reduces the cardiac dose even further: a prospective analysis. Radiat Oncol 2018; 13:116.

11. Andersen K, Vik-Mo H. Effects of spontaneous respiration on left ventricular function assessed by echocardiography. Circulation 1984;69:874-9.

12. Tomita H, Yamashiro T, Matsuoka S, Matsushita S, Kurihara $Y$, Nakajima Y. Changes in cross-sectional area and transverse diameter of the heart on inspiratory and expiratory chest CT: correlation with changes in lung size and influence on cardiotho- 
racic ratio measurement. PLOS One 2015;10:e0131902.

13. Pollom EL, Chin AL, Diehn M, Loo BW, Chang DT. Normal tissue constraints for abdominal and thoracic stereotactic body radiotherapy. Semin Radiat Oncol 2017;27:197-208.

14. Bae SH, Kim MS, Cho CK, et al. Predictor of severe gastroduodenal toxicity after stereotactic body radiotherapy for abdominopelvic malignancies. Int J Radiat Oncol Biol Phys 2012;84: e469-74.

15. Murphy JD, Christman-Skieller C, Kim J, Dieterich S, Chang DT, Koong AC. A dosimetric model of duodenal toxicity after stereotactic body radiotherapy for pancreatic cancer. Int J Radiat Oncol Biol Phys 2010;78:1420-6.

16. Kopek N, Holt MI, Hansen AT, Hoyer M. Stereotactic body radiotherapy for unresectable cholangiocarcinoma. Radiother Oncol 2010;94:47-52.

17. Goldsmith C, Price P, Cross T, Loughlin S, Cowley I, Plowman N. Dose-volume histogram analysis of stereotactic body radiothera- py treatment of pancreatic cancer: a focus on duodenal dose constraints. Semin Radiat Oncol 2016;26:149-56.

18. Kavanagh BD, Pan CC, Dawson LA, et al. Radiation dose-volume effects in the stomach and small bowel. Int J Radiat Oncol Biol Phys 2010;76(3 Suppl):S101-7.

19. Blanck O, Bode F, Gebhard M, et al. Dose-escalation study for cardiac radiosurgery in a porcine model. Int J Radiat Oncol Biol Phys 2014:89:590-8.

20. Hohmann S, Deisher AJ, Suzuki A, et al. Left ventricular function after noninvasive cardiac ablation using proton beam therapy in a porcine model. Heart Rhythm 2019;16:1710-9.

21. Bode $F$, Blanck 0 , Gebhard $M$, et al. Pulmonary vein isolation by radiosurgery: implications for non-invasive treatment of atrial fibrillation. Europace 2015;17:1868-74.

22. Gianni C, Rivera D, Burkhardt JD, et al. Stereotactic arrhythmia radioablation for refractory scar-related ventricular tachycardia. Heart Rhythm 2020;17:1241-8. 